\title{
Dynamics of Mitochondria and Mitochondrial $\mathrm{Ca}^{2+}$ near the Plasma Membrane of PC12 Cells
}

\section{A Study by Multimode Microscopy}

\author{
DE-MING YANG, ${ }^{a, f}$ CHUNG-CHIH LIN,${ }^{b, f, g}$ HSIA YU LIN,${ }^{c}$ \\ CHIEN-CHANG HUANG, ${ }^{d}$ DIN PING TSAI, ${ }^{c} \mathrm{CHIN}-\mathrm{WEN} \mathrm{CHI},{ }^{a}$ \\ AND LUNG-SEN KAO ${ }^{d, e, h}$
}

${ }^{a}$ Department of Medical Research and Education, Taipei Veterans General Hospital, Taipei, Republic of China

${ }^{b}$ Department of Life Sciences, Chung Shan Medical University, Taichung, Republic of China

${ }^{c}$ Department of Physics, National Taiwan University, Taipei, Republic of China

${ }^{d}$ Institute of Biochemistry, National Yang-Ming University, Taipei, Republic of China

${ }^{e}$ Department of Life Sciences, National Yang-Ming University, Taipei, Republic of China

\begin{abstract}
The goal of this study is to examine whether there is a difference in the regulation of $\mathrm{Ca}^{2+}$ between mitochondria near the cell surface and mitochondria in the cytosol. Total internal reflection fluorescence and epifluorescence microscopy were used to monitor changes in the mitochondrial $\mathrm{Ca}^{2+}$ $\left(\left[\mathrm{Ca}^{2+}\right]_{\mathrm{mt}}\right)$ between the mitochondria near the plasma membrane and those in the cytosol. The results show that $\left[\mathrm{Ca}^{2+}\right]_{\mathrm{mt}}$ near the plasma membrane increased earlier and decayed slower after high $\mathrm{K}^{+}$stimulation than average mitochondria in the cytosol. In addition, the changes in $\left[\mathrm{Ca}^{2+}\right]_{m t}$ in the mitochondria near the cell surface after a second stimulation were larger than those induced by the first stimulation. The results provide direct evidence to support the hypothesis that mitochondria in different subcellular localization show differential responses to the influx of extracellular $\mathrm{Ca}^{2+}$.
\end{abstract}

KEYWORDS: total internal reflection fluorescence microscopy; mitochondria; mitochondrial $\mathrm{Ca}^{2+} ; \mathrm{PC12}$ cells

\section{INTRODUCTION}

Mitochondria play multiple roles and these include generation of ATP, control of apoptosis, ${ }^{1}$ and buffering of cytosolic $\mathrm{Ca}^{2+}\left(\left[\mathrm{Ca}^{2+}\right]_{\mathrm{i}}\right){ }^{2}$ The spatial localization of mi-

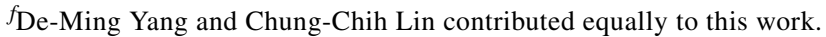

${ }^{g}$ Current address: Department of Life Sciences, National Yang-Ming University, Taipei, Taiwan, R.O.C.

${ }^{h}$ Also affiliated with Brain Research Center, University System of Taiwan, Taiwan, R.O.C.

Address for correspondence: Lung-Sen Kao, Ph.D., Department of Life Sciences, National Yang-Ming University, Taipei, Taiwan, R.O.C. Voice: +886-2-2826-7268; fax: +886-2-28234898.

1skao@ym.edu.tw
}

Ann. N.Y. Acad. Sci. 1042: 163-167 (2005). @ 2005 New York Academy of Sciences.

doi: 10.1196/annals.1338.018 
tochondria near endoplasmic reticulum or the plasma membrane has been proposed to be tightly associated with the homeostasis of $\left[\mathrm{Ca}^{2+}\right]_{\mathrm{i}}{ }^{3,4}$ However, there is very limited information available regarding differential changes in mitochondria located at different subcellular locations. In this study, we used total internal reflection (TIR) fluorescence microscopy, ${ }^{5,6}$ which detects only fluorescence near the plasma membrane; we combined this with conventional epifluorescence (EPI) microscopy, which detects fluorescence changes of total mitochondria population in a thicker focal plane, to monitor $\mathrm{Ca}^{2+}$ changes in mitochondria. By comparing the images of the same cell obtained alternately by TIR and EPI fluorescence microscopy, we were able to provide direct evidence that supports the hypothesis that mitochondria located near the cell surface respond differently from the total population of mitochondria to an influx of extracellular $\mathrm{Ca}^{2+}$.

\section{MATERIALS AND METHODS}

\section{Cell Culture}

Rat adrenal pheochromocytoma (PC12) cells were cultured as described previously. ${ }^{7,8}$

\section{Labeling of Fluorescence Indicators}

MitoTracker Green and rhod-2 were obtained from Molecular Probes (Eugene, OR). PC12 cells were incubated with $5 \mu \mathrm{M}$ of the acetoxymethyl esters form of MitoTracker Green for $15 \mathrm{~min}$ or $4.4 \mu \mathrm{M}$ rhod-2 for $45 \mathrm{~min}$ at $37^{\circ} \mathrm{C}$ in the loading buffer (for composition see Yang et al. ${ }^{7}$ ). PC12 cells were stimulated by high $\mathrm{K}^{+}$buffer as described previously. ${ }^{7}$

\section{Multimode Fluorescence Microscopy}

A dual condenser for TIR/EPI illumination (T.I.L.L. Photonics, Munich BioRegio, Germany) was added to a confocal laser scanning (CLS) microscopic system (FV300 upgrade; Olympus, Tokyo, Japan). A high numerical aperture objective $(\times 60$, PlanApo, N.A. $=1.45$, Olympus $)$ with the TIR condenser was used to achieve the TIR illumination. ${ }^{7}$ For CLS microscopic system, an Argon 488-nm laser (10 $\mathrm{mW}$ ) was used to excite MitoTracker Green, and a He-Ne 543-nm laser (1 mW) was used to excite rhod-2. The focal plane is controlled and locked by a Z-step motor on the CLS microscopic system. The confocal and differential interference contrast (DIC) images were acquired by a photomultiplier tube. Both TIR and EPI images were acquired alternately using a fast-cooled CCD (IMAGO QE, T.I.L.L. Photonics) and controlled by commercial software (TILLvisION 4.0, T.I.L.L. Photonics).

\section{RESULTS AND DISCUSSION}

\section{Morphology of Mitochondria}

The mitochondria of PC12 cells were stained by a specific fluorescence marker, MitoTracker Green (FIG. 1). Images of mitochondria of the same cell under CLS, 

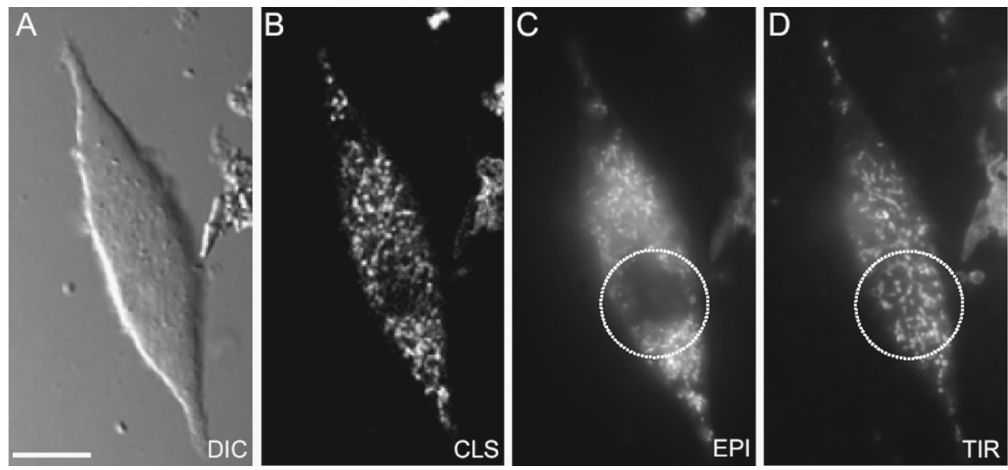

FIGURE 1. Images of mitochondria under a multimode microscope. PC12 cells were stained by MitoTracker Green. Images of the same cell under confocal laser scanning (CLS) mode (A), differential interference contrast (DIC) (B), epifluorescence (C), and total internal reflection (D) illumination are shown. Scale bar $=10 \mu \mathrm{m}$.

EPI, and TIR fluorescence microscopes were compared (FIG. 1). The morphology of mitochondria showed as dots and rod structures under the confocal and EPI modes, and more mitochondria showed a tubular form near the plasma membrane under the TIR mode. A better and clearer image of each mitochondrion could be obtained under the TIR and confocal modes. Mitochondria in some areas could not be detected (circled regions, FIG. 1C vs. 1D) under the EPI mode even though the optical section is thicker than that obtained under the TIR mode.

\section{Dynamics of Mitochondrial $\mathrm{Ca}^{2+}$ and Mitochondria near the Cell Surface}

Mitochondrial $\mathrm{Ca}^{2+}$ was monitored by a $\mathrm{Ca}^{2+}$ indicator rhod-2 that is known to be retained in the mitochondria. The confocal images of rhod-2 and MitoTracker Green were completely overlapped and are shown in Figure 2A. The results show that changes in rhod-2 fluorescence represent the changes in $\mathrm{Ca}^{2+}$ within the mitochondria. The microscope system allows time-dependent rhod-2 fluorescence changes to be alternately monitored by the TIR and EPI fluorescence modes and this approach was used. Changes in rhod-2 fluorescence of individual mitochondria under TIR illumination show that, after high $\mathrm{K}^{+}$stimulation, the rhod-2 fluorescence in the cytosol, but not that in the nucleus, increased immediately and remained high during the stimulation (FIG. 2C). To compare the mitochondria near the plasma membrane with those deep in the cytosol, we alternately recorded time-dependent changes of $\left[\mathrm{Ca}^{2+}\right]_{\mathrm{mt}}$ in the same cells under TIR and EPI illuminations (FIG. 2D). Rhod-2 fluorescence signals under TIR illumination were lower than those obtained under EPI illumination because the fluorescence collected from the evanescent field by TIR illumination is thinner than that from the focal plane by EPI illumination. The results show that TIR signals, which reflect changes in $\left[\mathrm{Ca}^{2+}\right]_{\mathrm{mt}}$ near the plasma membrane, increased earlier and decayed more slowly upon high $\mathrm{K}^{+}$stimulation than the EPI signals that represent the $\left[\mathrm{Ca}^{2+}\right]_{\mathrm{mt}}$ of the total mitochondria in a thicker focal plane. In addition, the changes in TIR fluorescence signal on a second stimu- 

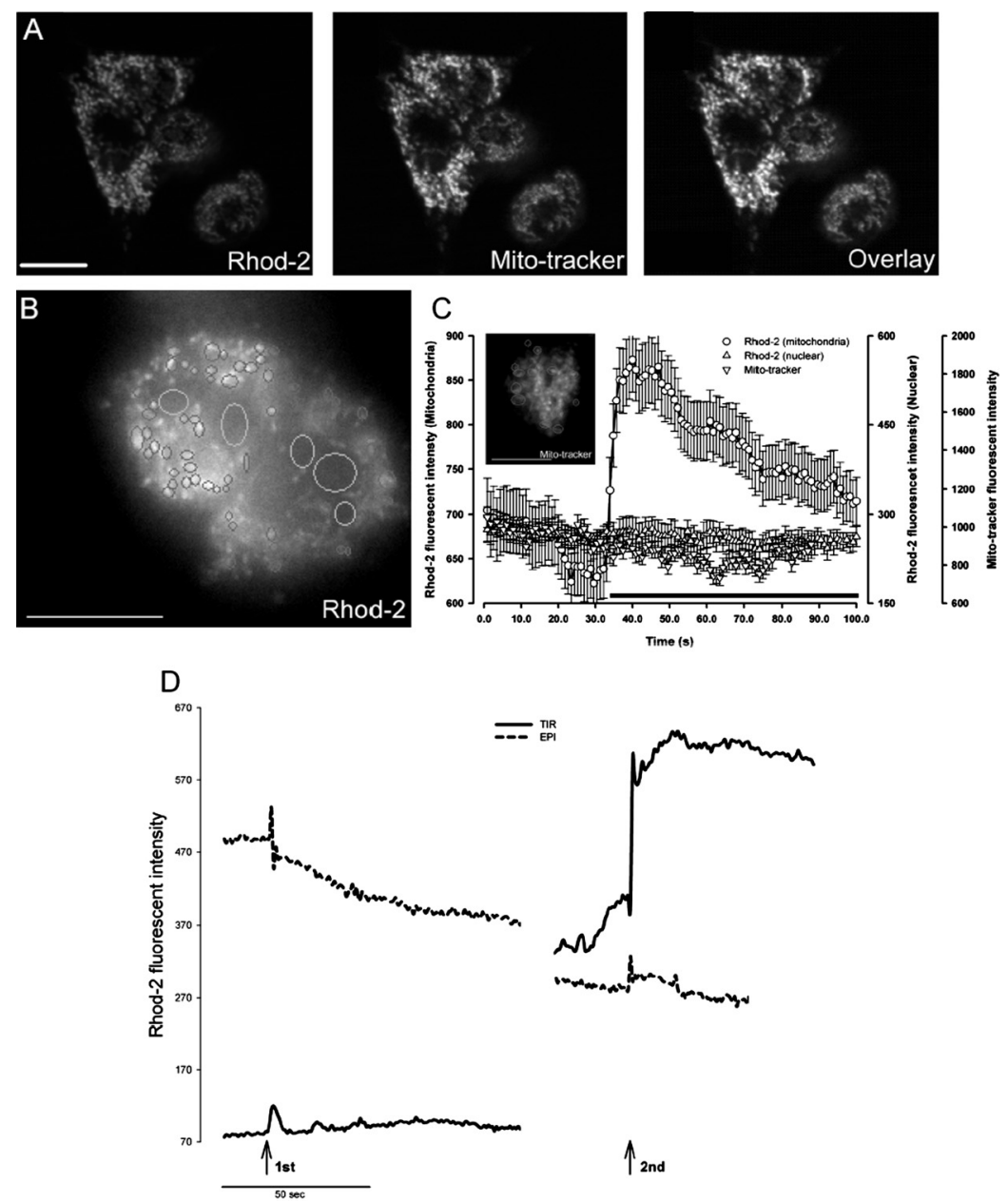

$\uparrow_{\text {2nd }}$

FIGURE 2. Time-dependent changes of $\left[\mathrm{Ca}^{2+}\right]_{\mathrm{mt}}$ in different subcellular location. (A) Confocal images of rhod-2, MitoTracker Green, and the overlaid images of PC12 cell. (B) TIR fluorescence image of rhod-2-stained PC12 cells. Mitochondria (57 filled circles) and regions in nuclei (5 open circles) were selected for analysis. As a negative control, a cell stained with MitoTracker Green was also analyzed similarly (19 circles). (C) Time-dependent changes of rhod-2 fluorescence in mitochondrial and nuclear regions. The black bar indicates the point of high $\mathrm{K}^{+}$stimulation. Data are mean \pm SEM of the fluorescence intensity of the areas selected in B. (D) Time-dependent changes of TIR (solid lines) and EPI fluorescence (dashed lines) signals. The cell received two stimulations. The second stimulation (2nd) was applied 5 min after the first one (1st). Arrows indicate where a 0.5 -s high $\mathrm{K}^{+}$ stimulation occurred. Scale bars $=10 \mu \mathrm{m}$. 
lation is larger and remained at a higher level for a longer time than those that occurred on the first stimulation. In contrast, the EPI fluorescence signals show that the fluorescence changes induced by the two stimulations were similar. It is possible that after the first stimulation, mitochondria near the plasma membrane continue to accumulate $\mathrm{Ca}^{2+}$ which leads to a higher $\left[\mathrm{Ca}^{2+}\right]_{\mathrm{mt}}$ level and saturates the pathways involved in maintaining the mitochondrial $\mathrm{Ca}^{2+}$ homeostasis; therefore, the subsequent stimulation induced a higher $\left[\mathrm{Ca}^{2+}\right]_{\mathrm{mt}}$ increase and remained at a plateau for a longer time. As a negative control, no changes in the fluorescence of MitoTracker were found upon high $\mathrm{K}^{+}$stimulation. This shows that the results indicate that the increase in rhod-2 fluorescence is not caused by the movement of mitochondria toward plasma membrane (FIG. 2C).

The results demonstrate that the response of mitochondria near the plasma membrane to the influx of extracellular $\mathrm{Ca}^{2+}$ was faster than the response of those located deep in the cytosol. It provides direct evidence to support the hypothesis that mitochondria near the plasma membrane are more active in taking up cytosolic $\mathrm{Ca}^{2+}$ than those deep in the cytosol. Finally, the detailed fluorescence images obtained using TIR illumination revealed the dynamics and heterogeneity of mitochondria in living cells.

\section{ACKNOWLEDGMENTS}

This research is supported by the grants from National Science Council (NSC-902112-M-002-047, NSC-90-2311-B-040-006), Taipei Veterans General Hospital (VGH92-385), Ministry of Education (Program for Promoting Academic Excellence of Universities, Grant 89-6-FA22-4), Brain Research Center, University System of Taiwan and Chung Shan Medical University (CSMU91-OM-B-014).

\section{REFERENCES}

1. Adrain, C. \& S.J. Martin. 2001. The mitochondrial apoptosome: a killer unleashed by the cytochrome seas. Trends Biochem. Sci. 26: 390-397.

2. CARAFOLI, E. 2003. Historical review: mitochondria and calcium: ups and downs of an unusual relationship. Trends Biochem. Sci. 28: 175-181.

3. Rizzuto, R. et al. 1998. Close contacts with endoplasmic reticulum as determinants of mitochondrial $\mathrm{Ca}^{2+}$ responses. Science 280: $1763-1766$.

4. YANG, D.-M. \& L.-S. KAO. 2001. Relative contribution of the $\mathrm{Na}^{+} / \mathrm{Ca}^{2+}$ exchanger, mitochondria and endoplasmic reticulum in the regulation of cytosolic $\mathrm{Ca}^{2+}$ and catecholamine secretion of bovine adrenal chromaffin cells. J. Neurochem. 76: 210 216.

5. AXELoRd, D. 1989. Total internal reflection fluorescence microscopy. Methods Cell Biol. 30: 245-270.

6. Stephens, D.J. \& V.J. Allan. 2003. Light microscopy techniques for living cell imaging. Science 300: $82-86$.

7. YANG, D.-M. et al. 2003. Tracking of secretory vesicles of PC12 cells using total internal reflection fluorescence microscopy. J. Microsc. 209: 223-227.

8. HuANG, C.-M. \& L.-S. KaO. 1996. Nerve growth factor, epidermal growth factor, and insulin differentially potentiate ATP-induced $\left[\mathrm{Ca}^{2+}\right]_{\mathrm{i}}$ rise and dopamine secretion in PC12 cells. J. Neurochem. 66: 124-130. 